\title{
Poor Adherence to Risk Stratification Guidelines Results in Overuse of Venous Thromboembolism Prophylaxis in Hospitalized Older Adults
}

\author{
Juliessa M. Pavon, MD, MHS ${ }^{1,2,3 *}$, Richard J. Sloane, MPH',2,3, Carl F. Pieper DrPH',2,3, Cathleen S. Colón-Emeric, MD, MHS 1,2,3, \\ Harvey J. Cohen, MD ${ }^{1,2,3}$, David Gallagher, MD', Miriam C. Morey, PhD',2,3, Midori McCarty', Thomas L. Ortel, MD PhD', \\ Susan N. Hastings, MD, MHS $1,2,3,4$
}

'Duke University, Durham, North Carolina; '2Geriatric Research Education Clinical Center, Durham Veteran Affairs Medical Center, Durham, North Carolina; ${ }^{3}$ Claude D. Pepper Older Americans Independence Center, Duke University, Durham, North Carolina; ${ }^{4}$ Health Services Research and Development, Durham Veterans Affairs Medical Center, Durham, North Carolina.

$\mathrm{V}$ enous thromboembolism (VTE) prophylaxis is an important consideration for every older adult admitted to the hospital ${ }^{1}$ but should not be prescribed to all patients. Use of anticoagulants (specifically low-molecular-weight heparin, low-dose unfractionated heparin, and fondaparinux) when not medically indicated may be harmful, especially for older adults who on average have more chronic conditions, ${ }^{1}$ take more potentially interacting medications, ${ }^{2}$ and have higher risks of bleeding. ${ }^{3}$ The American College of Chest Physicians (ACCP) Ninth Edition Guidelines for Antithrombotic Therapy and Prevention of Thrombosis explicitly recommend a risk-stratification approach using the Padua Prediction Score (PPS) to select those patients most likely to benefit from VTE prophylaxis. ${ }^{4,5}$ This study aimed to describe the use of risk stratification and pharmacologic VTE prophylaxis use in a population of medically ill, hospitalized older patients.

\section{METHODS}

We conducted a retrospective cohort study using data from patients aged 70 years or older admitted to Duke University Hospital general medicine services between January 1, 2014, to December 31, 2014. The PPS variables, 11 in total, are each weighed and sum to a score that stratifies patients into either high or low risk for VTE occurrence. ${ }^{5}$ Manual chart abstraction was performed using the electronic health record (EHR) to determine each patient's PPS, inpatient pharmacologic VTE prophylaxis use, and contraindications to VTE prophylaxis. Descriptive statistics are presented for the important confounders/covariates, VTE risk, and VTE prophylaxis use.

\section{RESULTS}

Of the total eligible cohort $(N=1,399), 400$ patients were randomly selected for manual chart review; 89 of these patients were not eligible because they were on anticoagulation upon

\footnotetext{
*Address for correspondence: Juliessa M. Pavon, MD, Duke University Health System, Division of Geriatrics, Box 3003, Durham, NC 27710; Telephone: 919684-8111; Fax: 919-668-0453; E-mail: Juliessa.pavon@duke.edu

Published online first February 6, 2018.
}

Received: July 7, 2017; Revised: October 2, 2017; Accepted: October 19, 2017 ๑ 2018 Society of Hospital Medicine DOI 10.12788/jhm.2916 admission, leaving $\mathrm{n}=311$ patients in the analytic sample. Mean age for the sample was 80.6 years (standard deviation [SD]: 7.3 ); $42 \%$ were male and $34 \%$ were African American, and median length of stay was 4.0 days. The overall mean PPS for the sample was 3.6 (SD 1.8), resulting in $59 \%(n=182)$ defined as "low risk." Reasons for admission, median length of stay, and aspirin use did not differ between the risk groups.

Pharmacological VTE prophylaxis was present in 74\% (134 out of 182) of low-risk patients and $71 \%$ (92 out of 129) of highrisk patients (Figure). In both low- and high-risk patients who received pharmacological VTE prophylaxis, over $90 \%$ had the therapy initiated within 24 hours of admission, and it was continued for over $60 \%$ of their hospital days.

\section{DISCUSSION}

We found no association between PPS and use of anticoagulants for VTE prophylaxis, suggesting that risk stratification is not being used to guide clinical decision-making. There are several barriers to implementing guideline directed use of VTE risk stratification. First, there is a lack of consensus on which VTE risk assessment tool is best to use with medically ill, hospitalized patients. While the ACCP Ninth Edition Guidelines support the use of the PPS, the American College of Physicians does not recommend a specific tool for VTE risk assessment. 5,6 Although other risk stratification tools exist, concordance between these tools has not been well studied. ${ }^{7}$ Second, manual calculation of the PPS can be cumbersome, error prone, and disruptive to the clinical workflow. Automated data extraction leveraging existing structured data elements in the EHR may be particularly attractive to many health systems striving to use EHRs to improve care. Designing and testing automatically populated VTE risk stratification tools may facilitate translation of evidence-based guidelines into routine clinical practice. Lastly, a key barrier is clinician education and awareness about these tools. Adding risk stratification tools to admission order sets is one way to increase clinician awareness and has been shown to decrease inappropriate VTE prophylaxis use. ${ }^{8}$ High-quality studies that use implementation science to promote uptake and efficacy of risk stratification tools into clinical practice are urgently needed.

Our study has several limitations. First, this was a single-site study at an academic center, which may limit generalizability of the findings. However, our design enabled us to look at other specific 


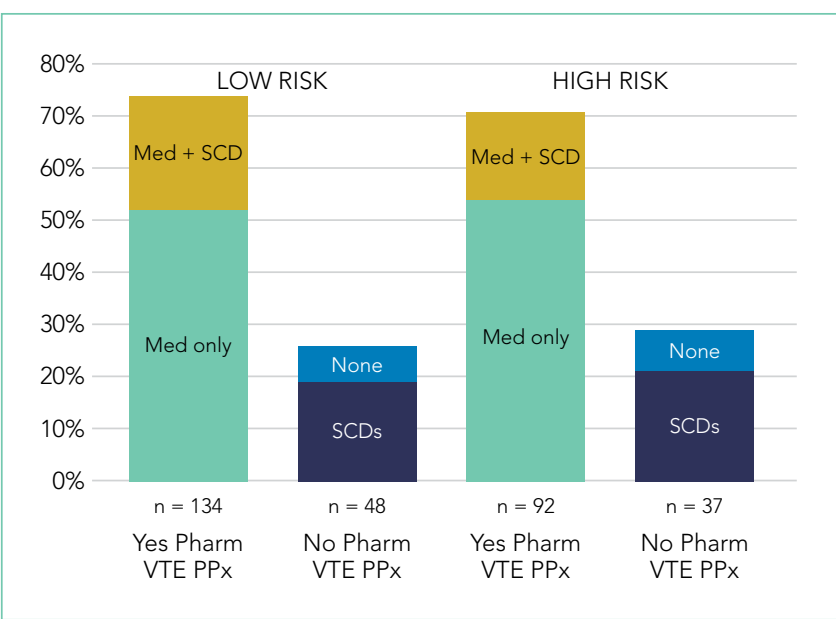

FIG. Indicates the percentage of low-risk and high-risk patients receiving pharmacological venous thromboembolism prophylaxis (either medication alone or in combination with sequential compression devices) or no pharmacological venous thromboembolism prophylaxis (either sequential compression devices only or no prophylaxis). Abbreviations: Med, medication; Pharm, pharmacologic; PPX, prophylaxis; SCD, sequential compression devices; VTE, venous thromboembolism.

patient-level data that is typically not available in larger databases. Second, determination of PPS is limited to data available in the $E H R$, resulting in measurement error and possibly the underreporting of risk factors. Finally, due to feasibility and the low probability of VTE, we did not collect data on long-term VTE outcome and were unable to determine the impact that inappropriate VTE prophylaxis use has in low-risk hospitalized older adults.

In summary, we found poor adherence to risk stratification guidelines among medically ill, hospitalized older adults, resulting in overuse of anticoagulants for VTE prophylaxis. Automating risk stratification tools and incorporating results into order sets may ensure that adequate prophylaxis is used for patients who need it, while minimizing excess prophylaxis in those who do not.

\section{Acknowledgments}

The authors would like to thank Shenglan Li from Research Triangle Institute for her assistance in the data programming and database creation.

Disclosure: The authors have no conflicts of interest to report. This study was funded by the National Institute on Aging (NIA) GEMSSTAR Award (NIA R03AG048007) and the Duke Older Americans Independence Center (NIA P30 AG028716-01). This work was also supported by the Duke University Internal Medicine Chair's Award, the Duke University Hartford Center of Excellence, and the Center of Innovation for Health Services Research in Primary Care (CIN 13-410) at the Durham VA Health Care System. This work was conducted while Dr. Pavon was supported by the T. Franklin Williams Scholars Program. Dr. Colón-Emeric is supported by K24 AG049077-01A1. The funding sources had no role in the design and conduct of the study; analysis or interpretation of the data; preparation or final approval of the manuscript before publication, and decision to submit the manuscript for publication. Disclaimer: The views expressed in this article are those of the authors and do not necessarily reflect the position or policy of Duke University or the Department of Veterans Affairs.

\section{References}

1. Kniffin WD, Baron JA, Barrett J, Birkmeyer JD, Anderson FA. The epidemiology of diagnosed pulmonary embolism and deep venous thrombosis in the elderly. Arch Intern Med. 1994;154(8):861-866.

2. Pasina L, Djade CD, Nobili A, et al. Drug-drug interactions in a cohort of hospitalized elderly patients. Pharmacoepidemiol Drug Saf. 2013; 22(10):1054-1060.

3. Campbell NR, Hull RD, Brant R, Hogan DB, Pineo GF, Raskob GE. Aging and heparin-related bleeding. Arch Intern Med. 1996;156(8):857-860.

4. Gould MK, Garcia DA, Wren SM, et al. Prevention of VTE in nonorthopedic surgical patients: Antithrombotic Therapy and Prevention of Thrombosis, 9th ed: American College of Chest Physicians Evidence-Based Clinical Practice Guidelines. Chest. 2012;141(2 Suppl):e227S-e277S.

5. Barbar S, Noventa F, Rossetto $V$, et al. A risk assessment model for the identification of hospitalized medical patients at risk for venous thromboembolism: the Padua Prediction Score. J Thromb Haemost. 2010;8(11):2450-2457.

6. Qaseem A, Chou R, Humphrey LL, Starkey M, Shekelle P. Venous thromboembolism prophylaxis in hospitalized patients: a clinical practice guideline from the American College of Physicians. Ann Intern Med. 2011;155(9):625-632.

7. Stuck AK, Spirk D, Schaudt J, Kucher N. Risk assessment models for venous thromboembolism in acutely ill medical patients. A systematic review. Thromb Haemost. 2017;117(4):801-808.

8. Khanna R, Vittinghoff E, Maselli J, Auerbach A. Unintended consequences of a standard admission order set on venous thromboembolism prophylaxis and patient outcomes. J Gen Intern Med. 2012;27(3):318-324. 